\title{
3D Wideband mmWave Localization for 5G Massive MIMO Systems
}

\author{
Zhipeng Lin ${ }^{\dagger *}$, Tiejun $\mathrm{Lv}^{\dagger}$, J. Andrew Zhang*, and Ren Ping Liu* \\ ${ }^{\dagger}$ School of Information and Communication Engineering \\ Beijing University of Posts and Telecommunications, Beijing, China \\ * School of Electrical and Data Engineering, University of Technology Sydney, Sydney, Australia \\ Email: \{linlzp, lvtiejun\}@ bupt.edu.cn, \{Andrew.Zhang, RenPing.Liu\}@uts.edu.au
}

\begin{abstract}
This paper proposes a novel 3D localization method for wideband mmWave massive MIMO systems. A high dimensional linear interpolation (HDLI)-based preprocessing is first proposed to transform the frequency-associated dynamical array response vectors into the common counterparts at the reference frequency. Through this method, the received data in all frequency bands can be processed jointly, and thus the high temporal resolution provided by wideband mmWave systems can be fully exploited for position estimation. To reduce the computational complexity in the process of the parameter estimation, we then present a wideband beamspace (WBS)based parameter estimation algorithm to estimate the angle and delay in the low-dimensional beamspace. By exploiting the quasi-optical propagation at the mmWave frequencies, a novel positioning scheme is also designed to determine the 3D location of the target. According to our analysis and simulation results, the proposed method is capable of achieving significantly reduced computational complexity, while maintaining high localization accuracy.
\end{abstract}

Index Terms-millimeter wave, massive MIMO, NLOS localization, angle and delay estimation

\section{INTRODUCTION}

Accurate localization of mobile devices is becoming increasingly important for many novel applications [1]. As a candidate technique for the fifth generation $(5 \mathrm{G})$ communication networks, millimeter-wave (mmWave) massive multipleinput multiple-output (MIMO) not only provides very high data rates and spectral efficiency, but also offers the possibility of precise positioning thanks to its high temporal resolution and high directivity [2].

Most of the existing positioning methods, e.g., [1], [3], [4], consider a narrowband channel model, and hence their algorithms could become ineffective in the practical systems where resolvable multipath signals in the temporal domain are present. Extension of these narrowband-based positioning methods to wideband systems is generally not straightforward, because channel parameters, e.g., signal subspace and steering matrices, actually vary with the carrier frequency [5]. Based on the assumption that the channel parameters remain constant in each narrowband channel, a widely used wideband treating method, i.e., incoherent signal-subspace processing (ISSP) [1], [6], [7], divides the wide frequency band into non-overlapping

The financial support of the National Natural Science Foundation of China (NSFC) (Grant No. 61671072) is gratefully acknowledged. narrowbands. This method runs positioning algorithms in each narrowband separately, and thus an additional processing needs be applied to combine all the results from individual narrowbands, resulting in an increase in computational complexity.

On the other hand, most of the positioning algorithms proposed for mmWave massive MIMO systems, such as [3], [4], [8], [9], assume that the line-of-sight (LOS) path exists between mobile station (MS) and base station (BS), and they only focused on two-dimension (2D) scenarios. In practice, the LOS path may not exist, especially in dense urban and indoor environments. In this case, both angle of arrival (AOA) and angle of departure (AOD) of each non-LOS (NLOS) path need be taken into consideration to achieve 3D localization.

In this paper, we propose a novel NLOS-based 3D localization method for wideband mmWave massive MIMO systems. Firstly, we propose a high dimensional linear interpolation (HDLI) as the preprocessing step to aggregate the information in all frequency bands. Then, a novel wideband beamspace (WBS)-based method is presented to estimate signal parameters in the beamspace instead of the original element space. As a result, the computational complexity can be dramatically reduced as the dimension of the original element space is very high. Finally, by exploiting the quasi-optical propagation at the mmWave frequencies, we propose a positioning strategy that estimates the 3D coordinates of the target MS. According to the complexity analysis and simulation results, our proposed method can achieve more accurate localization with less computational complexity, compared to the state-of-the-art technologies.

Notation: $a$, a and $\mathbf{A}$ stand for a scalar, a column vector, and a matrix, respectively; $\mathbf{I}_{K}$ represents a $K \times K$ identity matrix, and $\mathbf{0}_{M \times K}$ represents an $M \times K$ zero matrix; $\mathbf{1}_{K}$ denotes a vector of ones with a magnitude of $K ;[\mathbf{A}]_{i, j}$ is the entry on the $i$-th row and $j$-th column of $\mathbf{A} ;[\mathbf{A}]_{i, \text { : }}$ denotes the $i$-th row of $\mathbf{A}$; the inverse, transpose and conjugate transpose of $\mathbf{A}$ are represented by $\mathbf{A}^{-1}, \mathbf{A}^{T}$ and $\mathbf{A}^{H}$, respectively; $\|\mathbf{A}\|_{\mathrm{F}}$ and $\operatorname{vec}(\mathbf{A})$ denote the Frobenius norm and vectorization of $\mathbf{A}$, respectively; $\otimes, \oplus$ and $\diamond$ denote the Kronecker product, Kronecker sum, and Khatri-Rao product, respectively; expectation of a random variable is denoted by $\mathbb{E}\{\cdot\} ; O(\cdot)$ denotes the computational complexity. 


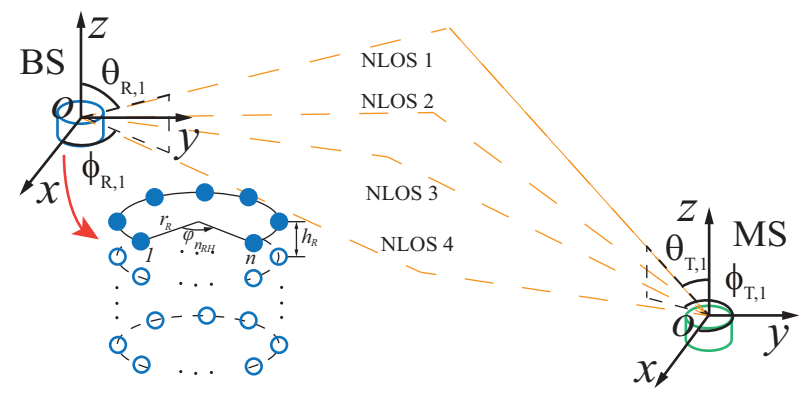

Fig. 1. 3D illustration positioning system for NLOS scenarios, where a BS is at a known position, and an MT needs to be located.

\section{System And Channel Models}

In dense urban and indoor propagation environments, the LOS propagation paths do not often exist. In this paper, we mainly study how to use NLOS path to realize 3D localization, as shown in Fig. 1. The positioning system includes a BS at a known position as the receiver, and an MS whose location is unknown and needs to be estimated. We consider a massive MIMO orthogonal frequency division multiplexing (OFDM) system with $N_{\mathrm{T}}$ transmit and $N_{\mathrm{R}}$ receive antennas. The received signal at subcarrier $n, n \in[0, N-1]$ is given by [10]

$$
\mathbf{y}[n]=\mathbf{H}[n] \mathbf{x}[n]+\mathbf{w}[n],
$$

where $\mathbf{x}[n] \in \mathbb{C}^{N_{\mathrm{T}} \times 1}$ and $\mathbf{w}[n] \in \mathbb{C}^{N_{\mathrm{R}} \times 1}$ represent the transmitted signal vector and the Gaussian noise associated with subcarrier $n$, respectively. $\mathbf{H}[n] \in \mathbb{C}^{N_{\mathrm{R}} \times N_{\mathrm{T}}}$ is the channel matrix, which can be expressed as

$$
\mathbf{H}[n]=\sum_{l=1}^{N_{\mathrm{p}}} \beta_{l} \mathbf{a}_{\mathrm{R}, n}\left(\phi_{\mathrm{R}, l}, \theta_{\mathrm{R}, l}\right) \mathbf{a}_{\mathrm{T}, n}^{H}\left(\phi_{\mathrm{T}, l}, \theta_{\mathrm{T}, l}\right) g_{n}\left(\tau_{l}\right)+\mathbf{n}[n] .
$$

In (2), $N_{\mathrm{p}}$ represents the number of received NLOS path$\mathrm{s}^{1} ; \mathbf{n}[n]$ is the zero-mean additive white Gaussian noise (AWGN); $\beta_{l}$ denotes the complex amplitude of the $l$-th path; $\mathbf{a}_{\mathrm{R}, n}\left(\phi_{\mathrm{R}, l}, \theta_{\mathrm{R}, l}\right)$ and $\mathbf{a}_{\mathrm{T}, n}\left(\phi_{\mathrm{T}, l}, \theta_{\mathrm{T}, l}\right)$ are the array response and steering vectors, respectively, with $\phi_{\mathrm{R}, l} / \theta_{\mathrm{R}, l}$ and $\phi_{\mathrm{T}, l} / \theta_{\mathrm{T}, l}$ being the azimuth/elevation of AOAs and AODs of the $l$-th path. $g_{n}\left(\tau_{l}\right)=e^{-j 2 \pi f_{n} \tau_{l}}$, where $\tau_{l}$ is the time delay of the $l$-th incoming path signal and $f_{n}$ is the frequency at the $n$-th subcarrier. $f_{n}=f_{0}+n \triangle_{\mathrm{F}}$, where $f_{0}$ is the carrier frequency at the lower end of the band and $\triangle_{\mathrm{F}}$ is the subcarrier spacing. It is noted that when the signal bandwidth is much smaller than the carrier frequency, then $f_{n} \approx f_{0}$ and (2) reverts to a standard narrowband channel model.

In our positioning system, for the simplicity of description, we assume that both BS and MS employ a uniform cylindrical array (UCyA). But the proposed scheme also works if MS uses a uniform rectangular array (URA). Fig. 1 only shows the geometric model of the UCyA employed in the BS for illustration. In the $\mathrm{UCyA}$, there are $N_{\mathrm{RV}}$ vertically arranged

\footnotetext{
${ }^{1}$ The LOS path can be regarded as a special case of NLOS.
}

UCAs, each of which consists of $N_{\mathrm{RH}}$ antennas horizontally, and the radius is $r_{\mathrm{R}}$. The vertical distance between any two adjacent UCAs is $h_{\mathrm{R}}$. The array response vector can be given by

$$
\mathbf{a}_{\mathrm{R}, n}\left(\phi_{\mathrm{R}, l}, \theta_{\mathrm{R}, l}\right)=\mathbf{a}_{\mathrm{RV}, n}\left(\theta_{\mathrm{R}, l}\right) \otimes \mathbf{a}_{\mathrm{RH}, n}\left(\phi_{\mathrm{R}, l}, \theta_{\mathrm{R}, l}\right),
$$

where

$$
\begin{aligned}
& {\left[\mathbf{a}_{\mathrm{RV}, n}\left(\theta_{\mathrm{R}, l}\right)\right]_{n_{\mathrm{RV}}, 1}} \\
& =\frac{1}{\sqrt{N_{\mathrm{RV}}}} \exp \left(-j \frac{2 \pi}{c} f_{n} h_{\mathrm{R}}\left(n_{\mathrm{RV}}-1\right) \cos \left(\theta_{\mathrm{R}, l}\right)\right)
\end{aligned}
$$

and

$$
\begin{aligned}
& {\left[\mathbf{a}_{\mathrm{RH}, n}\left(\phi_{\mathrm{R}, l}, \theta_{\mathrm{R}, l}\right)\right]_{n_{\mathrm{RH}}, 1}} \\
& =\frac{1}{\sqrt{N_{\mathrm{RH}}}} \exp \left(j \frac{2 \pi}{c} f_{n} r_{\mathrm{R}} \sin \left(\theta_{\mathrm{R}, l}\right) \cos \left(\phi_{\mathrm{R}, l}-\varphi_{n_{\mathrm{RH}}}\right)\right)
\end{aligned}
$$

are array response vectors in the vertical and horizontal plane, respectively. In the above equation, $\varphi_{n_{\mathrm{RH}}}=2 \pi\left(n_{\mathrm{RH}}-\right.$ 1) $/ N_{\mathrm{RH}}$ is the central angle difference between the $n_{\mathrm{RH}}$-th antenna and the first antenna of an UCA, as shown in Fig. 1. The array steering vector, $\mathbf{a}_{\mathrm{T}, n}\left(\phi_{\mathrm{T}, l}, \theta_{\mathrm{T}, l}\right)$, can be represented in a similar way.

\section{High DimENSIONAL LINEAR INTERPOLATION (HDLI)}

In this section, we propose an HDLI method to transform the dynamical response/steering vectors in (2) associated with the frequency $f_{n}$ into the common response/steering vectors at the reference frequency $f_{0}$. By using this method, the follow-on steps can process the information in all frequency bands jointly to fully exploit the high temporal resolution and the frequency diversity of the wideband OFDM system. We construct the virtual vertical and horizontal response vectors as

$$
\begin{aligned}
& {\left[\tilde{\mathbf{a}}_{\mathrm{RV}, n}\left(\theta_{\mathrm{R}, l}\right)\right]_{n_{\mathrm{RV}}, 1}} \\
& =\frac{1}{\sqrt{N_{\mathrm{RV}}}} \exp \left(-j \frac{2 \pi}{c} f_{n} h_{\mathrm{R}}[n]\left(n_{\mathrm{RV}}-1\right) \cos \left(\theta_{\mathrm{R}, l}\right)\right) \\
& =\left[\mathbf{a}_{\mathrm{RV}, 0}\left(\theta_{\mathrm{R}, l}\right)\right]_{n_{\mathrm{RV}}, 1}
\end{aligned}
$$

and

$$
\begin{aligned}
& {\left[\tilde{\mathbf{a}}_{\mathrm{RH}, n}\left(\phi_{\mathrm{R}, l}, \theta_{\mathrm{R}, l}\right)\right]_{n_{\mathrm{RH}}, 1}} \\
& =\frac{1}{\sqrt{N_{\mathrm{RH}}}} \exp \left(j \frac{2 \pi}{c} f_{n} r_{\mathrm{R}}[n] \sin \left(\theta_{\mathrm{R}, l}\right) \cos \left(\phi_{\mathrm{R}, l}-\varphi_{n_{\mathrm{RH}}}\right)\right) \\
& =\left[\mathbf{a}_{\mathrm{RH}, 0}\left(\phi_{\mathrm{R}, l}, \theta_{\mathrm{R}, l}\right)\right]_{n_{\mathrm{RH}}, 1}
\end{aligned}
$$

where $h_{\mathrm{R}}[n]$ and $r_{\mathrm{R}}[n]$ are the virtual vertical distance and radius depending on frequency $f_{n}$ [11]. Therefore, by sampling with intervals $h_{\mathrm{R}}[n]$ and $r_{\mathrm{R}}[n]$ at frequency $f_{n}$, the channel matrix in (2) will become the same for all frequencies. In fact, however, a perfect sampling by the Shannon-Whittaker interpolation [12] cannot be realized, and using $r_{\mathrm{R}}[n]$ as the sampling interval will make the channel matrix complex due to the nonlinear structure of UCAs. Here we propose an alternative approximation method to realize signal resampling. 
More precisely, applying the multidimensional interpolation to (2) leads to

$$
\begin{aligned}
& {\left[\tilde{\mathbf{H}}_{\mathrm{A}}[n]\right]_{n_{\mathrm{R}} \times n_{\mathrm{T}}}=[\mathbf{H}[n]]_{n_{\mathrm{R}} \times n_{\mathrm{T}}}+\frac{r_{\mathrm{R}}[n]}{r_{\mathrm{R}}} \triangle_{\mathbf{H}_{\mathrm{RH}}}} \\
& \quad+\frac{h_{\mathrm{R}}[n]}{h_{\mathrm{R}}} \triangle_{\mathbf{H}_{\mathrm{RV}}}+\frac{r_{\mathrm{T}}[n]}{r_{\mathrm{T}}} \triangle_{\mathbf{H}_{\mathrm{TH}}}+\frac{h_{\mathrm{T}}[n]}{h_{\mathrm{T}}} \triangle_{\mathbf{H}_{\mathrm{TV}}},
\end{aligned}
$$

where $\triangle_{\mathbf{H}_{\mathrm{RH}}}=[\mathbf{H}[n]]_{\left(n_{\mathrm{R}}+1\right) \times n_{\mathrm{T}}}-[\mathbf{H}[n]]_{n_{\mathrm{R}} \times n_{\mathrm{T}}}, \triangle_{\mathbf{H}_{\mathrm{RV}}}=$ $[\mathbf{H}[n]]_{\left(n_{\mathrm{R}}+N_{\mathrm{RH}}\right) \times n_{\mathrm{T}}}-[\mathbf{H}[n]]_{n_{\mathrm{R}} \times n_{\mathrm{T}}}, n_{\mathrm{R}}=N_{\mathrm{RH}}\left(n_{\mathrm{RV}}-1\right)+n_{\mathrm{RH}}$ and $n_{\mathrm{T}}=N_{\mathrm{TH}}\left(n_{\mathrm{TV}}-1\right)+n_{\mathrm{TH}} \cdot h_{\mathrm{T}} / h_{\mathrm{T}}[n]$ and $r_{\mathrm{T}} / r_{\mathrm{T}}[n]$ are the real/virtual vertical distance and radius of the transmit antenna, respectively. The matrices $\triangle_{\mathbf{H}_{\mathrm{TY}}}$ and $\triangle_{\mathbf{H}_{\mathrm{TX}}}$ can be constructed in a similar way.

\section{Positioning Estimation}

\section{A. WBS Transformation}

In this subsection, we firstly propose a WBS-based method before positioning estimation to reduce the computational complexity associated with the large dimension of the antenna array. Here we also use the process of transforming array response vectors as an example. By defining $p_{\mathrm{R}} \triangleq$ $-P_{\mathrm{R}},-P_{\mathrm{R}}+1, \ldots, P_{\mathrm{R}}$, we can construct a beamforming weight vector, $\mathbf{w}_{p_{\mathrm{R}}}$, as $\mathbf{w}_{p_{\mathrm{R}}}=\frac{1}{N_{\mathrm{RH}}}\left[e^{j p_{\mathrm{R}} \varphi_{1}}, \ldots, e^{j p_{\mathrm{R}} \varphi_{N_{\mathrm{RH}}}}\right]^{H}$. Then, the array pattern, $f_{p_{\mathrm{R}}, n_{\mathrm{RV}}}\left(\phi_{\mathrm{R}, l}, \theta_{\mathrm{R}, l}\right)$, can be written as

$$
f_{p_{\mathrm{R}}, n_{\mathrm{RV}}}\left(\phi_{\mathrm{R}, l}, \theta_{\mathrm{R}, l}\right)=\mathbf{w}_{p_{\mathrm{R}}}^{H} \mathbf{a}_{\mathrm{R}, 0, n_{\mathrm{RV}}}\left(\phi_{\mathrm{R}, l}, \theta_{\mathrm{R}, l}\right),
$$

where

$$
\mathbf{a}_{\mathrm{R}, 0, n_{\mathrm{RV}}}\left(\phi_{\mathrm{R}, l}, \theta_{\mathrm{R}, l}\right)=\left[\mathbf{a}_{\mathrm{RV}, 0}\left(\theta_{\mathrm{R}, l}\right)\right]_{n_{\mathrm{RV}}, 1} \cdot \mathbf{a}_{\mathrm{RH}, 0}\left(\phi_{\mathrm{R}, l}, \theta_{\mathrm{R}, l}\right) .
$$

The phase mode and the array pattern of the steering vectors, i.e., $p_{\mathrm{T}}$ and $f_{p_{\mathrm{T}}, n_{\mathrm{TV}}}\left(\phi_{\mathrm{T}, l}, \theta_{\mathrm{T}, l}\right)$, can be defined similarly. In accordance with the property of Kronecker product, i.e., $(\mathbf{A} \otimes \mathbf{B})(\mathbf{C} \otimes \mathbf{D})=\mathbf{A C} \otimes \mathbf{B D}$, we can transform the channel matrix into the beamspace as

$$
\begin{aligned}
& \overline{\mathbf{H}}[n]=\left(\mathbf{I}_{N_{\mathrm{RV}}} \otimes \mathbf{F}_{\mathrm{R}}\right) \tilde{\mathbf{H}}_{\mathrm{A}}[n]\left(\mathbf{I}_{N_{\mathrm{TV}}} \otimes \mathbf{F}_{\mathrm{T}}\right)^{T} \\
& =\sum_{l=1}^{N_{\mathrm{p}}} \beta_{l} \overline{\mathbf{a}}_{\mathrm{R}, 0}\left(\phi_{\mathrm{R}, l}, \theta_{\mathrm{R}, l}\right) \overline{\mathbf{a}}_{\mathrm{T}, 0}^{H}\left(\phi_{\mathrm{T}, l}, \theta_{\mathrm{T}, l}\right) g_{n}\left(\tau_{l}\right)+\overline{\mathbf{N}}[n],
\end{aligned}
$$

where $\mathbf{F}_{\mathrm{R}}=\sqrt{N_{\mathrm{RH}}}\left[j^{-P_{\mathrm{R}}} \mathbf{w}_{-P_{\mathrm{R}}}, \ldots, j^{P_{\mathrm{R}}} \mathbf{w}_{P_{\mathrm{R}}}\right]^{T}$ and $\mathbf{F}_{\mathrm{T}}=$ $\sqrt{N_{\mathrm{TH}}}\left[j^{-P_{\mathrm{T}}} \mathbf{w}_{-P_{\mathrm{T}}}, \ldots, j^{P_{\mathrm{T}}} \mathbf{w}_{P_{\mathrm{T}}}\right]^{T}$ are the beamforming matrices for the transmitter and receiver, respectively.

\section{B. Delay and Angle Estimation}

The transformed channel matrix $\overline{\mathbf{H}}[n]$ in (8) can be rewritten, in a more compact way, as

$$
\overline{\mathbf{H}}[n]=\overline{\mathbf{A}}_{\mathrm{R}} \Lambda[n] \overline{\mathbf{A}}_{\mathrm{T}}^{H}+\overline{\mathbf{N}}[n],
$$

where

$$
\overline{\mathbf{A}}_{\mathrm{R}}=\left[\overline{\mathbf{a}}_{\mathrm{R}, 0}\left(\phi_{\mathrm{R}, 1}, \theta_{\mathrm{R}, 1}\right), \ldots, \overline{\mathbf{a}}_{\mathrm{R}, 0}\left(\phi_{\mathrm{R}, N_{\mathrm{p}}}, \theta_{\mathrm{R}, N_{\mathrm{p}}}\right)\right] \in \mathbb{C}^{\bar{N}_{\mathrm{R}} \times N_{\mathrm{p}}}
$$

and

$$
\overline{\mathbf{A}}_{\mathrm{T}}=\left[\overline{\mathbf{a}}_{\mathrm{T}, 0}\left(\phi_{\mathrm{T}, 1}, \theta_{\mathrm{T}, 1}\right), \ldots, \overline{\mathbf{a}}_{\mathrm{T}, 0}\left(\phi_{\mathrm{T}, N_{\mathrm{p}}}, \theta_{\mathrm{T}, N_{\mathrm{p}}}\right)\right] \in \mathbb{C}^{\bar{N}_{\mathrm{T}} \times N_{\mathrm{p}}},
$$

contain the array response vectors for the transmitter and receiver after WBS preprocessing, with $\bar{N}_{\mathrm{R}}=N_{p_{\mathrm{R}}} N_{\mathrm{RV}}$ and
$N_{p_{\mathrm{R}}}=2 P_{\mathrm{R}}+1$, and $\Lambda[n]=\operatorname{diag}\left\{\beta_{1} g_{n}\left(\tau_{1}\right), \ldots, \beta_{N_{\mathrm{p}}} g_{n}\left(\tau_{N_{\mathrm{p}}}\right)\right\}$. Collecting channel matrices at all frequencies, we have

$$
\overline{\mathbf{H}}=[\overline{\mathbf{H}}[1], \overline{\mathbf{H}}[2], \ldots, \overline{\mathbf{H}}[N]] .
$$

Then we vectorize $\overline{\mathbf{H}}$ as

$$
\overline{\mathbf{h}}=\operatorname{vec}(\overline{\mathbf{H}}) \approx\left[\boldsymbol{\Gamma} \diamond \overline{\mathbf{A}}_{\mathrm{T}} \diamond \overline{\mathbf{A}}_{\mathrm{R}}\right] \mathbf{b}+\overline{\mathbf{n}}=\mathbf{U b}+\overline{\mathbf{n}},
$$

where $[\boldsymbol{\Gamma}]_{n, l}=g_{n}\left(\tau_{l}\right)$ and $\mathbf{b}=\left[\beta_{1}, \beta_{2}, \ldots, \beta_{N_{\mathrm{p}}}\right]^{T} . \mathbf{U} \in$ $\mathbb{C}^{M \times N_{\mathrm{p}}}$ is the space-time matrix, which is parametrized by the AOA, AOD and the path delays, and $M=N \bar{N}_{\mathrm{R}} \bar{N}_{\mathrm{T}}$. The covariance matrix of $\overline{\mathbf{h}}$ can be calculated as

$$
\mathbf{R}_{\overline{\mathbf{h}}}=\mathbb{E}\left\{\overline{\mathbf{h}} \overline{\mathbf{h}}^{H}\right\}=\mathbf{U} \boldsymbol{\Lambda}_{\mathbf{b}} \mathbf{U}^{H}+\sigma_{\mathrm{n}}^{2} \mathbf{I}_{M},
$$

where $\boldsymbol{\Lambda}_{\mathbf{b}}=\mathbb{E}\left\{\mathbf{b b}^{H}\right\}$ is a diagonal matrix. The eigenvaluedecomposition (EVD) of $\mathbf{R}_{\overline{\mathbf{h}}}$ can be obtained by

$$
\begin{aligned}
\mathbf{R}_{\overline{\mathbf{h}}} & =\left[\mathbf{E}_{\mathrm{s}}, \mathbf{E}_{\mathrm{n}}\right]\left[\begin{array}{cc}
\boldsymbol{\Sigma}_{\mathrm{s}} & \mathbf{0}_{N_{\mathrm{p}} \times\left(M-N_{\mathrm{p}}\right)} \\
\mathbf{0}_{\left(M-N_{\mathrm{p}}\right) \times N_{\mathrm{p}}} & \sigma_{\mathrm{n}}^{2} \mathbf{I}_{M-N_{\mathrm{p}}}
\end{array}\right]\left[\mathbf{E}_{\mathrm{s}}, \mathbf{E}_{\mathrm{n}}\right]^{H} \\
& =\mathbf{E}_{\mathrm{s}} \boldsymbol{\Sigma}_{\mathrm{s}} \mathbf{E}_{\mathrm{s}}^{H}+\sigma_{\mathrm{n}}^{2} \mathbf{E}_{\mathrm{n}} \mathbf{E}_{\mathrm{n}}^{H},
\end{aligned}
$$

where $\mathbf{E}_{\mathrm{s}} \in \mathbb{C}^{M \times N_{\mathrm{p}}}$ and $\mathbf{E}_{\mathrm{n}} \in \mathbb{C}^{M \times\left(M-N_{\mathrm{p}}\right)}$ correspond to the signal subspace and noise subspace, respectively, and $\boldsymbol{\Sigma}_{\mathrm{s}} \in$ $\mathbb{R}^{N_{\mathrm{p}} \times N_{\mathrm{p}}}$ is a diagonal matrix with diagonal elements being the largest $N_{\mathrm{p}}$ eigenvalues of $\mathbf{R}_{\overline{\mathbf{h}}}$. Based on $\mathbf{E}_{\mathrm{n}} \mathbf{E}_{\mathrm{n}}^{H}+\mathbf{E}_{\mathrm{s}} \mathbf{E}_{\mathrm{s}}^{H}=$ $\mathbf{I}_{N_{\mathrm{p}}}$, (13) is rewritten as

$$
\mathbf{R}_{\overline{\mathbf{h}}}=\mathbf{E}_{\mathrm{s}}\left(\boldsymbol{\Sigma}_{\mathrm{s}}-\sigma_{\mathrm{n}}^{2} \mathbf{I}_{N_{\mathrm{p}}}\right) \mathbf{E}_{\mathrm{s}}^{H}+\sigma_{\mathrm{n}}^{2} \mathbf{I}_{N_{\mathrm{p}}} .
$$

According to (12), (14), we can obtain

$$
\mathbf{E}_{\mathrm{s}}=\mathbf{U T}
$$

where $\mathbf{T}$ is a full rank matrix.

1) Delay Estimation: In order to obtain the delay-related submatrix, $\mathbf{U}_{\mathrm{D}}$, from the space-time matrix, $\mathbf{U}$, we define the delay-selection matrix as $\mathbf{J}_{\mathrm{D}}=\operatorname{diag}\left(\mathbf{J}_{\mathrm{D}, 1}, \ldots, \mathbf{J}_{\mathrm{D}, N}\right)$, where $\mathbf{J}_{\mathrm{D}, n}=\mathbf{1}_{\bar{N}_{\mathrm{T}} \bar{N}_{\mathrm{R}}}^{T}$. Then we have $\mathbf{U}_{\mathrm{D}}=\mathbf{J}_{\mathrm{D}} \mathbf{U}$. By defining $\tilde{\mathbf{J}}_{\mathrm{D}, n}=\left[\mathbf{0}_{1 \times(n-1)}, 1, \mathbf{0}_{1 \times(N-n)}\right]$, the delay-related submatrix associated with subcarrier $n$ can be calculated as $\mathbf{U}_{\mathrm{D}, n}=$ $\tilde{\mathbf{J}}_{\mathrm{D}, n} \mathbf{U}_{\mathrm{D}}$. Thus, we can obtain a linear recurrence relation between the delay-related submatrices of each frequency:

$$
\mathbf{U}_{\mathrm{D}, \tilde{n}+1}=\mathbf{U}_{\mathrm{D}, \tilde{n}} \boldsymbol{\Theta}_{\mathrm{D}},
$$

where $\Theta_{\mathrm{D}}=\operatorname{diag}\left(e^{-j 2 \pi \triangle_{\mathrm{f}} \tau_{1}}, \ldots, e^{-j 2 \pi \triangle_{\mathrm{f}} \tau_{N_{\mathrm{p}}}}\right)$ and $\tilde{n}=$ $1,2, \ldots N-1$. Accoring to (15), the delay-related submatrix of the signal subspace matrix at frequency, $f_{n}$, can be calculated as

$$
\mathbf{E}_{\mathrm{D}, n}=\tilde{\mathbf{J}}_{\mathrm{D}, n} \mathbf{J}_{\mathrm{D}} \mathbf{E}_{\mathrm{s}}=\mathbf{U}_{\mathrm{D}, n} \mathbf{T} .
$$

Substituting (16) into (17), we obtain

$$
\mathbf{E}_{\mathrm{D}, \tilde{n}+1}=\mathbf{E}_{\mathrm{D}, \tilde{n}} \mathbf{T}^{-1} \boldsymbol{\Theta}_{\mathrm{D}} \mathbf{T}=\mathbf{E}_{\mathrm{D}, \tilde{n}} \mathbf{\Psi}_{\mathrm{D}}
$$

By using the total least-squares (TLS) criterion, we can estimate $\Psi_{\mathrm{D}}=\mathbf{T}^{-1} \boldsymbol{\Theta}_{\mathrm{D}} \mathbf{T}$ as $\hat{\mathbf{\Psi}}_{\mathrm{D}, \tilde{n}}$, each of which has in total $N_{\mathrm{p}}$ sorted eigenvalues, i.e., $\lambda_{\mathrm{D}, \tilde{n}, N_{\mathrm{p}}}$. Due to the fact that the eigenvalues of an upper triangular matrix are also the diagonal elements of this matrix, we can obtain $N-1$ different 
estimates for each $\Theta_{\mathrm{D}}$. As a result, the delay, $\tau_{n_{\mathrm{p}}}$, of the $n_{\mathrm{p}}$-th path can be estimated as

$$
\hat{\tau}_{l}=\frac{1}{N-1} \sum_{\tilde{n}}^{N-1}\left[\frac{j \ln \left(\lambda_{\mathrm{D}, \tilde{n}, l}\right)}{2 \pi \triangle_{\mathrm{F}}}\right] .
$$

2) Angle Estimation: The processes of selecting the AOA/AOD-related submatrices are similar to that of selecting the delay-related submatrix. We define the AOA selection matrices as $\mathbf{J}_{\mathrm{R}}=\mathbf{1}_{\bar{N}_{\mathrm{T}} N}^{T} \otimes \mathbf{I}_{\bar{N}_{\mathrm{R}}}$. Then the AOA-related submatrices can be formulated as $\mathbf{U}_{\mathrm{R}}=\mathbf{J}_{\mathrm{R}} \mathbf{U}$. The linear recurrence relation between the beamspace array response matrices of each pair of UCAs is $\mathbf{U}_{\mathrm{RV}, \tilde{n}_{\mathrm{RV}}+1}=\mathbf{U}_{\mathrm{RV}, \tilde{n}_{\mathrm{RV}}} \boldsymbol{\Theta}_{\mathrm{R}}$, where $\Theta_{R}$ is a diagonal matrix, whose element is $\left[\boldsymbol{\Theta}_{\mathrm{R}}\right]_{l, l}=e^{-j \frac{2 \pi}{c} f_{0} h \cos \left(\theta_{\mathrm{R}, l}\right)}$, and $\tilde{n}_{\mathrm{RV}}=1,2, \ldots, N_{\mathrm{RV}}-1$. $\mathbf{U}_{\mathrm{RV}, \tilde{n}_{\mathrm{RV}}}=\mathbf{J}_{\mathrm{RV}, n_{\mathrm{RV}}} \mathbf{U}_{\mathrm{R}} \in \mathbb{C}^{N_{p_{\mathrm{R}}} \times N_{\mathrm{p}}}$ is a submatrix of $\mathbf{U}_{\mathrm{R}}$, where $\quad \mathbf{J}_{\mathrm{RV}, n_{\mathrm{RV}}}=\left[\mathbf{0}_{N_{p_{\mathrm{R}}} \times N_{p_{\mathrm{R}}}\left(n_{\mathrm{RV}}-1\right)}, \mathbf{I}_{N_{p_{\mathrm{R}}}}, \mathbf{0}_{N_{p_{\mathrm{R}}} \times N_{p_{\mathrm{R}}}\left(N_{\mathrm{RV}}-1\right)}\right]$. Using the similar method as that used in delay estimation, the elevation AOA of the $l$-th path, $\hat{\theta}_{\mathrm{R}, l}$, is estimated as

$$
\hat{\theta}_{\mathrm{R}, l}=\frac{1}{N_{\mathrm{RV}}-1} \sum_{\tilde{n}_{\mathrm{RV}}}^{N_{\mathrm{RV}}-1}\left[\arccos \left(\frac{j c \ln \left(\lambda_{\mathrm{R}, \tilde{n}_{\mathrm{RV}}, l}\right)}{2 \pi f_{0} h}\right)\right],
$$

where $\lambda_{\mathrm{R}, \tilde{n}_{\mathrm{RV}}, n_{\mathrm{p}}}$ is the $l$-th eigenvalue of $\hat{\Psi}_{\mathrm{D}, \tilde{n}}$, and $\hat{\mathbf{\Psi}}_{\mathrm{D}, \tilde{n}}$ is the estimated matrix of $\Psi_{\mathrm{R}}=\mathbf{T}^{-1} \mathbf{\Theta}_{\mathrm{R}} \mathbf{T}$.

As an alternative to multiparameter pair matching algorithms [9] which generally have very high complexity, we use the estimated values of the elevation AOAs to estimate their related azimuth AOAs. Similar to (15), we con construct the signal subspace, $\mathbf{E}_{\mathrm{S}_{\mathrm{RV}, n_{\mathrm{RV}}}}$, and the noise subspace, $\mathbf{E}_{\mathrm{n}_{\mathrm{RV}, n_{\mathrm{RV}}}}$, of the $n_{\mathrm{RV}}$-th UCA, and the azimuth AOA of the $l$-th path can be estimated by

$$
\hat{\phi}_{\mathrm{R}, l}=\arg \max _{\Phi_{\mathrm{R}}} \frac{1}{\left\|\mathbf{E}_{\mathrm{n}_{\mathrm{RV}, n_{\mathrm{RV}}}^{H}} \overline{\mathbf{a}}_{\mathrm{RV}, n_{\mathrm{RV}}}\left(\Phi_{\mathrm{R}}, \hat{\theta}_{\mathrm{R}, l}\right)\right\|_{\mathrm{F}}^{2}},
$$

where

$$
\left[\overline{\mathbf{a}}_{\mathrm{RV}, n_{\mathrm{RV}}}\left(\Phi_{\mathrm{R}}, \theta_{\mathrm{R}, l}\right)\right]_{p_{\mathrm{R}}, 1}=\sqrt{N_{\mathrm{RH}}} j^{-p_{\mathrm{R}}} f_{p_{\mathrm{R}}, n_{\mathrm{RV}}}\left(\Phi_{\mathrm{R}}, \theta_{\mathrm{R}, l}\right) .
$$

$\Phi_{\mathrm{R}}$ is the azimuth of the AOA, which can be estimated by 1D search. Then we can use the similar methods to estimate the azimuth/elevation of AODs.

Due to the present of noise, the estimated channel parameters of each path cannot be matched automatically. Thus, pair matching operation needs to be done here, and we use a simplified perturbation term-based method [13] to realize parameter matching. In our localization scheme, the estimated values of elevation AOA/AOD are used for the estimation of the azimuth AOA/AOD, so the azimuth and elevation angles of each path can be matched automatically. As for the pair matching of delay and angle, we can use the delay of each path as the benchmark and add perturbation matrices, $P_{\mathrm{R}} / P_{\mathrm{T}}$, to the eigenvalue matrices of elevation $\mathrm{AOA} / \mathrm{AOD}, \Psi_{\mathrm{R}} / \Psi_{\mathrm{T}}$, to mute the impact of noise. Using the elevation of AOA as an example, $P_{\mathrm{R}}$ can be calculated by

$$
\operatorname{vec}\left(P_{\mathrm{R}}\right)=\left[\boldsymbol{\Psi}_{\mathrm{D}}^{T} \oplus-\boldsymbol{\Psi}_{\mathrm{D}}\right]^{\dagger} \operatorname{vec}\left(\boldsymbol{\Psi}_{\mathrm{D}} \boldsymbol{\Psi}_{\mathrm{R}}-\boldsymbol{\Psi}_{\mathrm{R}} \boldsymbol{\Psi}_{\mathrm{D}}\right)
$$

where $\Psi_{\mathrm{D}}$ is the delay eigenvalue matrices defined in (18).

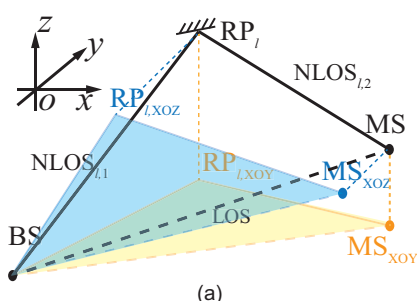

(a)

Fig. 2. The geometrical relationship between BS and MS. (a) Stereo view; (b) Projection on XOY plane; (c) Projection on XOZ plane.

\section{Positioning strategy}

After obtaining the delay and the azimuth/elevation of AOAs and AODs of each path by using the above mentioned approaches, in this subsection we estimate the MS position by exploiting the quasi-optical propagation at the mmWave frequencies. In practice, when the signal operating at mmWave frequencies propagates and reflects off a surfacs, it fades much more quickly than those at lower frequencies [14]. Thus, it is a reasonable assumption that almost all of the NLOS paths we receive at BS are single-bounce reflections. To illustrate position estimation, in this subsection we use the $l$-th NLOS path reflected from the $l$-th reflection point $\left(\mathrm{RP}_{l}\right)$ as an example. The total length of the $l$-th NLOS path is $d_{l}=c\left(\hat{\tau}_{l}+\tau_{\text {of }}\right)$, where $\tau_{\text {of }}$ is the time offset between BS and MS. We divide this path into two parts, $\operatorname{NLOS}_{l, 1}$ and $\operatorname{NLOS}_{l, 2}$, as shown in Fig. 2(a), i.e., $d_{l}=d_{l, 1}+d_{l, 2}$, where $d_{l, 1}$ and $d_{l, 2}$, are the lengths of $\operatorname{NLOS}_{l, 1}$ and $\operatorname{NLOS}_{l, 2}$. The black dashed line in Fig. 2(a) is the virtual LOS path between BS and MS, whose length is $d_{0}$. We can see BS, $\mathrm{RP}_{l}$ and MS form a triangle, and its projections on the XOY and XOZ planes are painted yellow and blue, respectively. $\mathrm{RP}_{l, \mathrm{XOY}}, \mathrm{RP}_{l, \mathrm{XOZ}}$, $\mathrm{MS}_{\mathrm{XOY}}$, and $\mathrm{MS}_{\mathrm{XOZ}}$ are the projections of $\mathrm{RP}_{l}$ and $\mathrm{MS}$ on the XOY and XOZ planes, respectively.

According to the geometrical relationship projected on the XOY plane, as shown in Fig. 2(b), we construct the following equations

$$
\left\{\begin{array}{l}
\cos \left(\hat{\phi}_{\mathrm{T}, l}-\hat{\phi}_{\mathrm{R}, l}\right)=\frac{d_{0, \mathrm{XOY}}^{2}-d_{l, 1, \mathrm{XOY}}^{2}-d_{l, 2, \mathrm{XOY}}^{2}}{2 d_{l, 1, \mathrm{XOY}} d_{l, \mathrm{XOY}, 2}}, \\
d_{0, \mathrm{XOY}} \cos \phi_{0}=d_{l, 1, \mathrm{XOY}} \cos \hat{\phi}_{\mathrm{R}, l}-d_{l, 2, \mathrm{XOY}} \cos \hat{\phi}_{\mathrm{T}, l},
\end{array}\right.
$$

where $d_{0, \mathrm{XOY}}=d_{0} \sin \theta_{0}, d_{l, 1, \mathrm{XOY}}=d_{l, 1} \sin \hat{\theta}_{\mathrm{R}, l}$, and $d_{l, 2, \mathrm{XOY}}=d_{l, 2} \sin \hat{\theta}_{\mathrm{T}, l}$ are the lengths of the projections of LOS, $\operatorname{NLOS}_{l, 1}$, and $\operatorname{NLOS}_{l, 2}$ on the XOY plane, and $\phi_{0}$ and $\theta_{0}$ are the azimuth and the elevation of AOA of the LOS path. Similar to Fig. 2(b), we can also construct the equations based on the geometrical relationship shown in Fig. 2(c)

$$
\left\{\begin{array}{l}
\cos \left(\hat{\theta}_{\mathrm{R}, l, \mathrm{XOZ}}+\hat{\theta}_{\mathrm{T}, l, \mathrm{Xoz}}\right)=\frac{d_{0, \mathrm{xoz}}^{2}-d_{l, 1, \mathrm{xoz}}^{2}-d_{l, 2, \mathrm{xoz}}^{2}}{2 d_{l, 1, \mathrm{Xoz}} d_{l, 2, \mathrm{Xoz}}}, \\
d_{0} \cos \theta_{0}=d_{l, 1} \cos \hat{\theta}_{\mathrm{R}, l}-d_{l, 2} \cos \hat{\theta}_{\mathrm{T}, l},
\end{array}\right.
$$

where $d_{0, \mathrm{XOZ}}=d_{0}\left(1-\sin ^{2} \phi_{0} \sin ^{2} \theta_{0}\right)^{1 / 2}, d_{l, 1, \mathrm{XOZ}}=d_{l, 1}(1$ $\left.-\sin ^{2} \hat{\phi}_{\mathrm{R}, l} \sin ^{2} \hat{\theta}_{\mathrm{R}, l}\right)^{1 / 2}$, and $d_{l, 2, \mathrm{XOZ}}=d_{l, 2}\left(1-\sin ^{2} \hat{\phi}_{\mathrm{T}, l}\right.$ 
$\left.\sin ^{2} \hat{\theta}_{\mathrm{T}, l}\right)^{1 / 2}$ are the lengths of the projections of LOS, $\mathrm{NLOS}_{l, 1}$, and $\mathrm{NLOS}_{l, 2}$ on the XOZ plane, respectively; $\hat{\theta}_{\mathrm{R}, l, \mathrm{XOZ}}=\arctan \left(\tan \hat{\theta}_{\mathrm{R}, l} \cos \hat{\phi}_{\mathrm{R}, l}\right)$ and $\hat{\theta}_{\mathrm{T}, l, \mathrm{XOZ}}=$ $\arctan \left(-\tan \hat{\theta}_{\mathrm{T}, l} \cos \hat{\phi}_{\mathrm{T}, l}\right)$ are the angles between the positive $\mathrm{z}$-axis and the projections of AOA and AOD of the $l$-th path on the XOZ plane, respectively. By solving (23) and (24), we can obtain the estimated value of $d_{0}, \phi_{0}$ and $\theta_{0}$, i.e., $\hat{d}_{0}, \hat{\phi}_{0}$ and $\hat{\theta}_{0}$, and the estimated MS position related to the $l$-th path can be calculated as

$$
\left\{\begin{array}{l}
\hat{x}_{l}=\hat{d}_{0} \sin \hat{\theta}_{0} \cos \hat{\phi}_{0} \\
\hat{y}_{l}=\hat{d}_{0} \sin \hat{\theta}_{0} \sin \hat{\phi}_{0} \\
\hat{z}_{l}=\hat{d}_{0} \cos \hat{\theta}_{0} .
\end{array}\right.
$$

By using the similar methods to deal with the estimated parameters of all the received paths, we can obtain in total $N_{\mathrm{p}}$ solutions $^{2}$. In order to further reduce the estimation error, we calculate the mean of these solutions to obtian the final position of $\mathrm{MS}^{3}$.

\section{Complexity Analysis}

In this subsection, we discuss the computational complexity of the proposed localization method and compare it with that of the quadric rotational invariance property-based method (QRIPM) [16] and the generalized beamspace-based method (GBM) [1]. We assume that the numbers of antennas deployed in BS and MS are large enough. Because QRIPM and GBM use the ISSP method to process wideband signals, in the process of angle and delay estimation, the computational complexity of them is $O\left(N_{\mathrm{T}}^{3} N_{\mathrm{R}}^{3} N^{4}\right)$ and $O\left(\bar{N}_{\mathrm{T}}^{3} \bar{N}_{\mathrm{R}}^{3} N_{\mathrm{p}}^{2} N^{2}\right)$, respectively, while the complexity of the proposed method is only $O\left(\bar{N}_{\mathrm{T}}^{3} \bar{N}_{\mathrm{R}}^{3} N^{3}\right)$.

As for the pair matching operation, traditional multiparameter estimation algorithms mainly use the exhaustive search (ES)-based method to match all possible pairs of angles. This method will lead to extremely high computational complexity, if the number of estimated parameters is large. Because our positioning problem needs to match five parameters for each path, the computational complexity of traditional pair matching methods, e.g., the minimum eigenvalue spectrum parameter matching [16], is $O\left(N^{2} M^{5} N_{\mathrm{p}}^{2} \sum_{l=1}^{N_{\mathrm{p}}} l^{5}\right)$. Comparatively, the computational complexity of our proposed method is only $O\left(N_{\mathrm{p}}^{3}\right)$.

\section{Simulation Results}

In this section, we present MATLAB simulation results to demonstrate the performance of the proposed algorithm. We set $f_{0}=30 \mathrm{GHz}$ and $B=2 \mathrm{GHz}$, and assume that there are in total $N_{\mathrm{p}}=10$ NLOS paths and $N=20$ consecutive subcarriers. The number of transmitting antennas is set to

\footnotetext{
${ }^{2}$ The time offset, $\tau_{\text {of }}$, is an unknown parameter independent of the transmission path. This parameter can be calculated by assuming that two of the estimated NLOS paths can be converged at a point.

${ }^{3}$ It is noted that in practice, diffuse scatterings and higher-order bounces can also be received by BS but they are much less than the single bounces. In this case, we can treat all of the received paths as the single-bounce paths and then use clustering algorithms [15] to eliminate the unexpected results before computing the mean.
}

$N_{\mathrm{T}}=10$. The distance, $h_{\mathrm{R}}$, between adjacent receiving UCAs and the radius, $r_{\mathrm{R}}$, of UCyA are $0.5 \lambda_{0}$ and $2 \lambda_{0}$, respectively. We calculate the root mean square error (RMSE) over all the trials to evaluate the performance of angle and delay estimation, and the proposed algorithm is also compared with QRIPM [16] and GBM [1]. Due to space limitations, this paper only provides the results for AOA and delay estimation, and AOD estimation results are similar to AOA's.

Figs. 3(a)-(c) show the RMSE of the estimated azimuth and elevation of AOAs and the delays versus the number of receive antennas under different signal-to-noise ratio (SNR) conditions. In Fig. 3(a), we can see that the RMSE of the estimated azimuth of AOAs decreases as the average received SNR or the number of receive antenna increases. It can also be observed that when the number of antennas is small, the RMSE performance of GBM and the proposed method is worse than that of QRIPM. This is because that in the beamspace-based methods, the array pattern in (7) contains a residual term, which is non-integrable and can be neglected only when the antennas is far more than the phase modes, i.e., $N_{\mathrm{R}} \gg N_{p_{\mathrm{R}}}$ [1]. However, when the number of antennas increases, the RMSE performance of GBM and the proposed method decreases faster than that of QRIPM, and our method achieves better performance than the other two methods. The estimated elevation of AOAs is shown in Fig. 3(b). We can see that when the number of receive antennas is less than 100 , the beamspace-based methods still cannot achieve the performance as good as QRIPM. However, our proposed method performs best when the number of antennas is large. In addition, compared with the results shown in Fig. 3(a), we can see that the estimates of the elevation of AOAs are less affected by the beamspace transformation than that of the azimuth of AOAs. It can be explained that on the vertical dimension, the response vectors of each UCA in UCyA have undergone the similar beamspace transformation. On the other hand, it can be seen in Fig. 3(c) that our method outperforms the other two in the process of delay estimation. The reason is that by using the proposed positioning estimation methods, the high temporal resolution provided by wideband mmWave systems can be fully exploited. However, Fig. 3(c) also shows that the delay precision is little influenced by the increased number of receive antennas, because its precision mainly depends on the transmission bandwidth.

We also characterize the performance of the algorithms by plotting the cumulative distribution function (CDF) of the localization error, as shown in Fig. (4). We observe that while the performance of the proposed method decreases with the average received SNR decreasing, the localization error statistics remain good even for $\mathrm{SNR}=-5 \mathrm{~dB}$ : in this case, the probability of achieving a sub-meter localization error can approach to 1 . In addition, for high SNR values, such as $10 \mathrm{~dB}$, even when the number of receive antenna is 150 , the probability of achieving a centimeter-level localization accuracy is larger than 0.5 . 

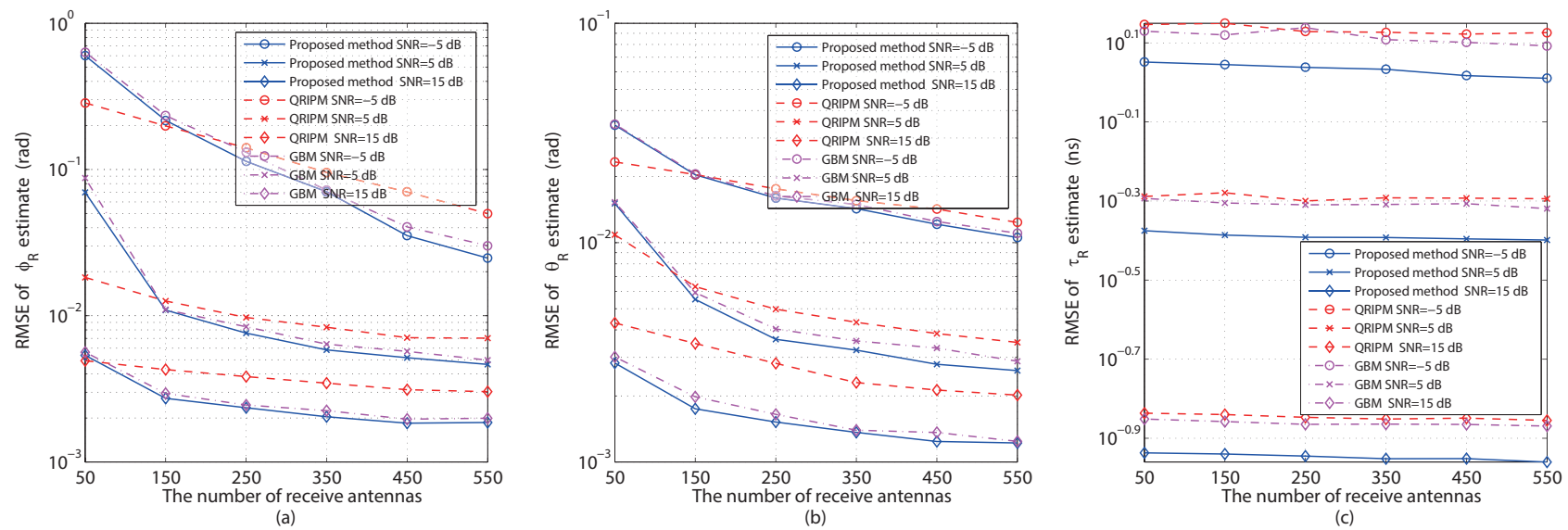

Fig. 3. Comparison of the RMSE performance vs. the number of BS antennas for the estimation of different parameters by the different estimation methods. (a) Azimuth of AoA; (b) Elevation of AoA; (c) Path delay.

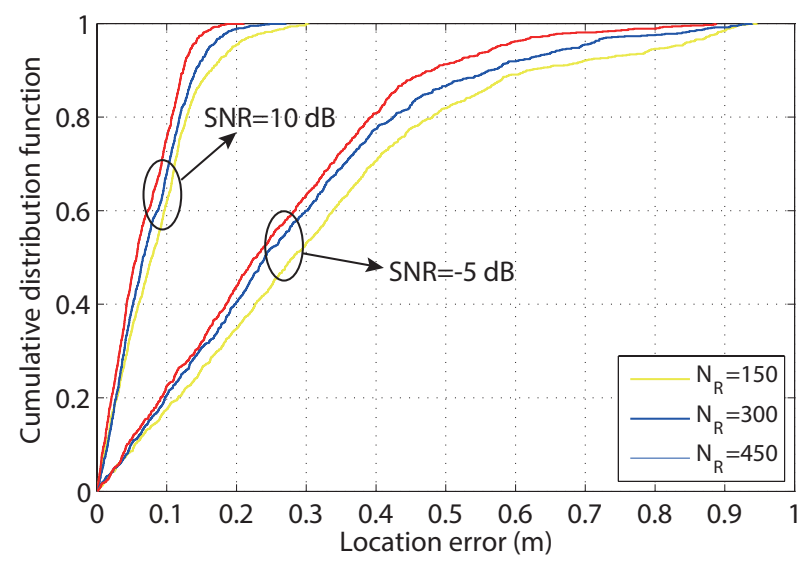

Fig. 4. CDF of the localization error for different numbers of receive antennas under different conditions of received SNR.

\section{CONCLUSIONS}

In this paper, a novel NLOS-based localization method has been proposed for wideband mmWave massive MIMO systems. To enable joint information processing at all frequencies for the wideband signal, a preprocessing step based on high dimensional linear interpolation was designed firstly. Then we presented a wideband-based positioning algorithm to realize low-complexity 3D localization. Simulation results show that when a large number of antennas is deployed, our proposed method is capable of precisely estimating the angle and delay even at very low SNR values. Potential applications of this work include solving the localization problems in $5 \mathrm{G}$ vehicle to vehicle $(\mathrm{V} 2 \mathrm{~V})$ networks, joint communication and radar sensing (JCAS) networks, and so on.

\section{REFERENCES}

[1] Z. Lin, T. Lv, and P. T. Mathiopoulos, "3-D indoor positioning for millimeter-Wave massive MIMO systems," IEEE Trans. Commun., vol. 66, no. 6, pp. 2472-2486, June 2018.

[2] N. Garcia, H. Wymeersch, E. G. Larsson, et al., "Direct localization for massive MIMO," IEEE Trans. Signal Process., vol. 65, no. 10, pp. 2475-2487, May 2017.
[3] N. Amiot, T. Pedersen, M. Laaraiedh, et al., "A hybrid positioning method based on hypothesis testing," IEEE Wireless Commun. Lett., vol. 1, no. 4, pp. 348-351, Aug. 2012.

[4] A. Shahmansoori, G. E. Garcia, and G. Destino, "5G position and orientation estimation through millimeter wave MIMO," in Proc. IEEE Globecom Workshops (GC Wkshps), San Diego, CA, Dec. 2015, pp. $1-6$.

[5] R. J. Mailloux, Phased Array Antenna Handbook, Artech House, Norwood, United States, 2nd edition edition, 2005.

[6] B. D. Van Veen and K. M. Buckley, "Beamforming: A versatile approach to spatial filtering," IEEE Acoust. Speech Sig. Proc. Mag., vol. 5, no. 5, pp. 4-24, Apr. 1988.

[7] F. Raimondi, P. Comon, and O. Michel, "Wideband multilinear array processing through tensor decomposition," in Proc. IEEE Int. Conf. Acoust. Speech Signal Process. (ICASSP), Shanghai, China, Mar. 2016, pp. 2951-2955.

[8] M. Vari and D. Cassioli, "mmWaves RSSI indoor network localization," in Proc. IEEE Int. Conf. on Commun. Workshop (ICCW), Sydney, Australia, Jun. 2014, pp. 127-132.

[9] A. Hu, T. Lv, H. Gao, Z. Zhang, and S. Yang, "An ESPRIT-based approach for 2-D localization of incoherently distributed sources in massive MIMO systems," IEEE J. Sel. Topics Signal Process., vol. 8, no. 5, pp. 996-1011, Oct. 2014.

[10] A. Shahmansoori, G. E. Garcia, and G. Destino, "Position and orientation estimation through millimeter-wave mimo in $5 \mathrm{~g}$ systems," Digit. Signal Process., vol. 17, no. 3, pp. 1822-1835, Mar. 2018.

[11] J. Zhang, I. Podkurkov, M. Haardt, et al., "Efficient multidimensional parameter estimation for joint wideband radar and communication systems based on OFDM," in Proc. IEEE Int. Conf. Acoust. Speech Signal Process. (ICASSP), New Orleans, LA, USA, Mar. 2017, pp. 3091-3100.

[12] C. E. Shannon, "Communication in the presence of noise," Proceedings of the IRE, vol. 37, no. 1, pp. 10-21, Jan. 1949.

[13] A. J. van der Veen, P.B. Ober, and E.F. Deprettere, "Azimuth and elevation computation in high resolution doa estimation," IEEE Trans. Signal Process., vol. 40, no. 7, pp. 1828-1832, July 1992.

[14] Mathew K. Samimi and Theodore S. Rappaport, "3-D millimeter-wave statistical channel model for 5G wireless system design," IEEE Trans. Microw. Theory Techn., vol. 64, no. 7, pp. 2207-2225, July 2016.

[15] M. Ester, H.-P. Kriegel, and J. Sander, "Spatial data mining: A database approach," in Proc. 5th Int. Symp. Large Spatial Databases (SSD), Berlin, Germany, Jul. 1997, pp. 47-66.

[16] X. Guo, Q. Wan, X. Shen, and H. Dou, "Low-complexity parameters estimator for multiple 2D domain incoherently distributed sources," Turk. J. Elect. Eng. Comput. Sci., vol. 3, no. 19, pp. 445-462, May 2011. 